\title{
Enhanced UV Emission of GaN Nanowires Functionalized by Wider Bandgap Solution-Processed p-MnO Quantum Dots
}

Dhaifallah Almalawi; †, "l,\# Sergei Lopatin; ${ }^{\S}$ Somak Mitra; ${ }^{\dagger}$ Tahani Flemban; ${ }^{\perp}$ AlexandraMadalina Siladie, $;$ Bruno Gayral, $;$ Bruno Daudin, $;$ and Iman S. Roqan ${ }^{\dagger, \#, *}$

$\dagger$ Physical Sciences and Engineering Division, King Abdullah University of Science and Technology (KAUST), Thuwal 23955-6900, Saudi Arabia.

$\$$ University of Grenoble-Alpes, CEA-IRIG, PHELIQS, 17 av. des Martyrs, F-38000, Grenoble, France.

$\S$ Imaging and Characterization Laboratory, King Abdullah University of Science and Technology (KAUST), Thuwal 23955-6900, Saudi Arabia.

${ }^{\perp}$ Department of Physics, College of Science, Imam Abdulrahman Bin Faisal University (IAU), Dammam 31441, Saudi Arabia.

" Physics Department, Faculty of Science, Taif University, 21974 Taif, P. O. Box 888, Saudi Arabia.

*Email:iman.roqan@kaust.edu.sa

\# Equal contribution authors

Keywords: III-nitrides, semiconductor Nanowires, light emitting devices, p-type MnO quantum dots, electron energy loss spectroscopy.

\begin{abstract}
GaN-based UV light emitting devices suffer from low efficiency. To mitigate this issue, we hybridized GaN nanowires (NWs) grown on Si substrates by plasma-assisted molecular beam
\end{abstract}


epitaxy with solution-processed p-type $\mathrm{MnO}$ quantum dots (QDs) characterized by a wider bandgap $(\sim 5 \mathrm{eV})$ than that of GaN. Further investigations reveal that the photoluminescence intensity of the GaN NWs increases up to $\sim 3.9$-fold ( $290 \%)$ after functionalizing them with p$\mathrm{MnO}$ QDs, while the internal quantum efficiency is improved by $\sim 1.7$-fold. Electron energy loss spectroscopy (EELS) incorporated into transmission electron microscopy (TEM) reveals an increase in the density of states in QD-decorated NWs compared to the bare ones. The advanced optical and EELS analyses indicate that the energy transfer from the wider-bandgap p-MnO QDs to $\mathrm{n}-\mathrm{GaN}$ NW leads to substantial emission enhancement and a greater radiative recombination contribution, due to the good band alignment between MnO QDs and GaN NW. This work provides valuable insight into an environmentally-friendly strategy for improving UV device performance. 


\section{Introduction}

Technologies based on GaN and related III-nitrides are widely used in several applications, such as light emitting diodes (LEDs), laser diodes (LDs), photodetectors, and high-power and high-frequency devices, ${ }^{1,2}$ owing to the unique electrical and optical properties of these materials, such as direct bandgap, wide spectral tunability, good conductivity, and durability in harsh environments. In particular, GaN-based UV LEDs have been extensively studied, with the view of utilizing them in curing, medical devices, and diverse industrial domains. ${ }^{3-5}$ However, the UV GaN-based LED performance is significantly below that of the devices emitting in the visible spectral range, due primarily to low external efficiency $(<10 \%)$ at the emission wavelengths below $365 \mathrm{~nm} .{ }^{6,7}$ The low internal quantum efficiency (IQE) is one of the issues that undermine the external efficiency as a result of high threading dislocation (TD) density caused by lattice mismatch with commonly utilized substrates, which introduces high density of non-radiative centers. $^{5,6}$ Therefore, developing a UV LED structure that can yield the required efficiency remains highly challenging.

Recently, GaN nanowires (NWs) have emerged as promising candidates for enhancing the optical efficiency of UV LEDs and reducing the TD effects. Indeed, their large surface-to-volume ratio promotes an elastic strain relaxation mechanism, thus preventing the formation of dislocations. ${ }^{7,8}$ Moreover, NW structures, which are inherently diffusive, permit light extraction efficiency enhancement. ${ }^{9}$ However, the surface states characterizing NWs can lead to nonradiative recombination, which can markedly reduce optical efficiency. In this case, Fermi level pinning effects, along with the enhanced non-radiative recombination rate due to the presence of the surface defects (which form trapping centers), reduce LED efficiency due to the Shockley-Read-Hall (SRH) non-radiative recombination defect. ${ }^{5,10}$ This issue can be partly 
mitigated by NW surface passivation using complex chemicals. ${ }^{5,11}$ However, this method relies on highly toxic solutions, such as diluted potassium hydroxide $(\mathrm{KOH})$ solution, for the removal of unwanted surface defects from III-nitride nanowires. ${ }^{5}$ Available evidence indicates that inhalation of vapor produced by such solutions can cause serious damage to the upper respiratory tract and the mucous membranes. This method reduces the trap states only, resulting in insufficient enhancement (less than two folds enhancement). ${ }^{5}$ In addition, when the $\mathrm{KOH}$ passivation methods are used, $<100 \%$ improvement in the UV emission of GaN NWs can be attained. Thus, there is still a need for a method that is environmentally friendly and is capable of producing greater improvements in the GaN-based NW UV emission, while being cost-effective for large-scale applications. ${ }^{5,} 12$ This method reduces the trap states only, resulting in insufficient enhancement (less than two folds enhancement). ${ }^{5}$ Thus, more effective alternatives are still needed, significant efforts were being dedicated to the improvement of $\mathrm{GaN}$ emission, which would in turn result in efficient UV LEDs. ${ }^{13,14}$

QD-functionalized Si NW for solar cell applications has been used to enhanced light absorption, however, such design has not been used to enhance the mission emitted from III-nitride based emitting devices. ${ }^{15}$ Recently, we explored new wide-bandgap ( $\left.>4.5 \mathrm{eV}\right)$ crystalline p-type manganese oxide quantum dots (p-MnO QDs) synthesized by solution-processed femtosecond laser ablation technique in liquid (FLAL). ${ }^{16,17}$ However, the effect of GaN NWs hybridized with wider-bandgap $\mathrm{MnO}(\sim 4.8 \mathrm{eV}$, located in the deep UV spectral region) have not yet been investigated.

These gaps in extant knowledge have prompted this investigation, as a part of which a novel strategy based on hybridizing p-type MnO QDs with GaN by simple and cost-effective dropcasting method was adopted to improve the optical GaN efficiency through energy transfer from 
QDs to GaN. The obtained findings demonstrate that functionalizing GaN NWs by solutionprocessed p-MnO QDs is effective in enhancing the UV optical efficiency. This study included advanced optical characterizations and analyses, as well as electron energy loss spectroscopy (EELS) measurements, all of which indicated that the energy transfer (ET) can occur from the QDs to GaN, resulting in significant UV emission enhancement. Hence, this investigation provides valuable insight into the UV emitting device development. The novelty of our work stems from using wider-bandgap QDs to enhance the III-nitride UV and deep UV emission significantly, which is a method that has not been investigated. In addition, the optical properties of solution processed p-MnO QD-functionalized III-nitrides have not been investigated as well.

\section{Results and Discussions}

As a part of our previous work focusing on the properties of solution-processed p-type MnO QDs and their synthesis, ${ }^{17} \mathrm{MnO}$ QDs were synthesized by cost-effective FLAL at room temperature (RT) and under ambient conditions. The FLAL synthesis of MnO QDs was carried out using Ti:sapphire Coherent Mira 900 laser (pulse width 150 fs, $800 \mathrm{~nm}$ wavelength, operating at $76 \mathrm{MHz}$ frequency) that was focused on the $\mathrm{MnO}$ target in ethanol solution. ${ }^{17}$

The GaN NWs were grown along the c-axis by catalyst-free, plasma-assisted molecular beam epitaxy (PA-MBE) in a MECA2000 MBE apparatus. The substrates were 2-inch wide Si (111) oriented wafers, which were de-oxidized in hydrofluoric acid prior to their introduction into the MBE chamber. The NWs were grown under nitrogen-rich conditions with a radio frequency power of $300 \mathrm{~W}$ for the plasma cell and a $\mathrm{N}_{2}$ flux of 0.6 standard cubic centimeters per minute (sccm). The $\mathrm{Ga} /$ active $\mathrm{N}_{2}$ flux ratio was typically equal to 0.3 . A thin $(\sim 3 \mathrm{~nm})$ AlN buffer layer was deposited on Si prior to the GaN NW growth to prevent the in-plane tilt and twist of the wires and to homogenize the NW density across the entire Si wafer surface. 
NW material morphology was characterized by scanning electron microscopy (SEM, FEI Nova Nano 630). Moreover, high resolution transmission electron microscopy (HR-TEM) and scanning TEM (STEM), as well as ultrahigh-resolution EELS measurements, were performed to study QD-decorated NWs structures and to measure the bandgap differences between bare and QD-decorated GaN NWs. STEM imaging or spectrum imaging (SI) measurements were performed at $80 \mathrm{kV}$ using Titan Themis Z TEM (Thermo Fisher, formerly known as FEI Co, USA) operating in the 40-300 kV range, which was equipped with a double Cs (spherical aberration) corrector, a high brightness electron gun (x-FEG), an electron beam monochromator, and a Gatan Quantum 966 imaging filter (GIF). The low-loss spectra were acquired in the so-called microprobe STEM mode with about $1 \mathrm{mrad}$ semi-convergence angle (4 $\mathrm{nm}$ probe size). The electron beam monochromator operation was optimized by adopting the method first implemented by Govyadinov and colleagues ${ }^{18}$ and described in detail by Lopatin et al. ${ }^{19}$ UV-vis Varian Cary 5000 spectrophotometer was employed to evaluate the GaN NW bandgap through RT absorption measurements. Material optical properties before and after QD drop-casting on NWs were examined by conducting RT micro-photoluminescence ( $\mu$-PL) measurements using Horiba LabRAM Aramis Raman spectrometer attached to Kimmon Koha continuous-wave (CW) He-Cd laser $(\lambda=325 \mathrm{~nm})$ and $40 \times$ objective. PL measurements before and after QD drop-casting on NWs were performed at $5 \mathrm{~K}$ and RT by a $325 \mathrm{~nm} \mathrm{CW} \mathrm{He}-\mathrm{Cd}$ laser and a $244 \mathrm{~nm}$ (the double frequency of the $488 \mathrm{~nm}$ line) $\mathrm{Ar}^{+}$LEXEL laser, whereby PL signals were captured by an Andor monochromator connected to a charge-coupled device camera. In addition, to obtain RT PL excitation (PLE) spectra, Edinburgh Instruments FLS980 spectrometer attached to a $1000 \mathrm{~W}$ Xe lamp (Newport) was used. Note that no PL measurements were acquired within the first 30 minutes 
after drop-casting the solution-processed MnO QDs on GaN NWs, as our prior examinations indicated that this was sufficient for ensuring that ethanol has evaporated completely.

The main image and inset in Figure 1a show cross-sectional and top SEM view of one of the GaN NW samples grown on Si substrate, revealing their average length of $\sim 750 \mathrm{~nm}$ and $\sim 35$ $\mathrm{nm}$ average diameter, while the other GaN NW sample is characterized by a $\sim 3.5 \mu \mathrm{m}$ and $\sim 50 \mathrm{~nm}$ average length and diameter, respectively. The HR-TEM image shown in Figure 1b indicates that the solution-processed p-MnO QDs are highly crystalline and their diameter ranges from 3.5 to 7 nm. The STEM image of a single GaN NW decorated by MnO QDs through the drop-casting method is shown in Figure 1c. (note that Figure 1c suggest that some QDs attached to the NWs are clustered) According to our previously reported XPS findings, the MnO phase is dominant $(81.5 \%)$ in the composition of these $\mathrm{p}-\mathrm{MnO}$ QDs, with much smaller contributions from $\mathrm{MnOOH}$ $(12.0 \%)$ and $\mathrm{Mn}_{2} \mathrm{O}_{3}(6.5 \%)$, resulting in the unique characteristics discussed in our previous work. ${ }^{17,20}$ As a part of the same study, we demonstrated their p-type conductivity using field effect transistor (FET) and Kelvin probe measurements. ${ }^{17}$
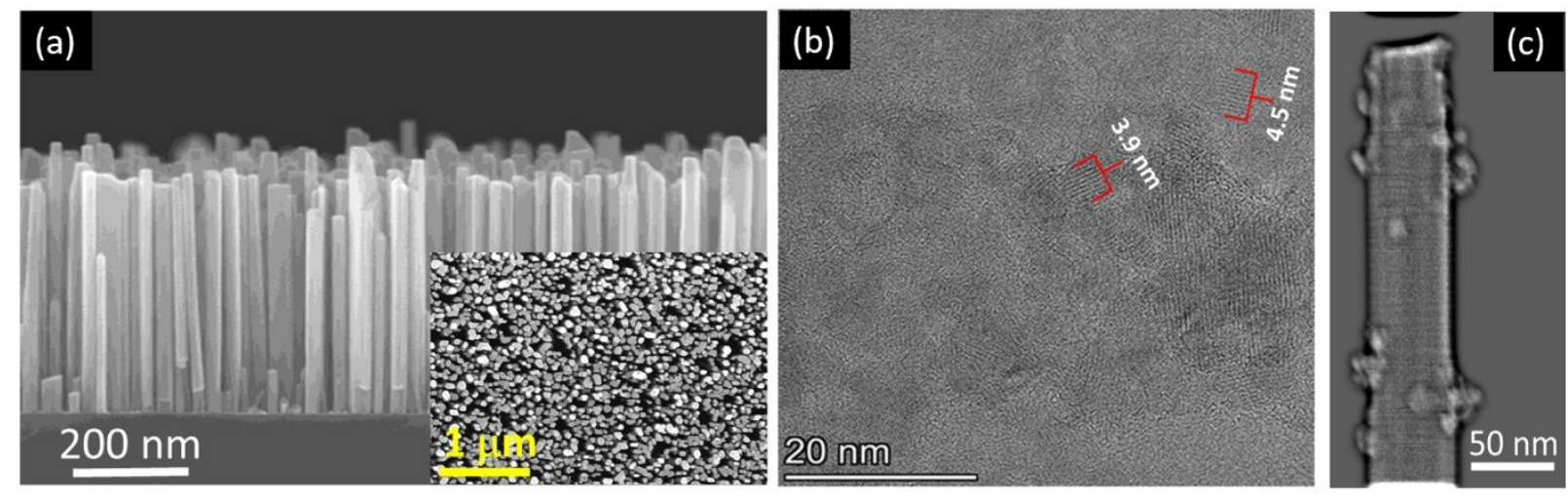

Figure 1. (a) Cross-sectional SEM image of GaN NWs (with the top view shown in the inset). (b) HR-TEM images of MnO QDs in a TEM grid. (c) STEM image of MnO QD-decorated GaN NWs (The image is obtained using a monochromated electron beam with a very small convergence angle to improve the EELS energy resolution). 
To determine the bandgap of the p-MnO QDs, absorption and PLE measurements were performed at RT. Figure $2 \mathrm{~b}$ shows the corrected PL emission from the p-MnO QDs. The corrected PLE and absorption spectra of $\mathrm{MnO}$ QDs shown in Figure 2a confirm that their bandgap ( $\sim \mathrm{eV})$ is wider than that of $\mathrm{GaN}(3.5 \mathrm{eV})$, which is in line with our previously reported findings. ${ }^{17} \mathrm{~A}$ slight tail shown in the 4.65-4.3 eV (265-285 $\mathrm{nm})$ range can be related to surface states in QDs, as discussed in our recent reports. $\mathrm{S}^{16,17}$ It should be noted that both the absorption and the PLE spectra have been corrected by subtracting the ethanol signal (the PL and PLE spectra of ethanol are provided in Figure S1 in Supporting Information).

To study the effect of $\mathrm{MnO}$ QD functionalization on GaN emission, we investigated the PL and $\mu$-PL emission produced by GaN before and after functionalizing NWs with QDs. Figure 2c shows the GaN near band edge emission (NBE) at $363.5 \mathrm{~nm}(3.41 \mathrm{eV})$ before and after $\mathrm{MnO}$ QD drop-casting at RT, whereby the samples were excited by $244 \mathrm{~nm}$ (corresponding to the energy above the MnO bandgap), with the $\mu$-PL emission obtained by exciting the samples by $325 \mathrm{~nm}$ presented in the inset. Notably, no MnO emission from QD-decorated NWs was observed even when the samples were excited by $\lambda=244 \mathrm{~nm}$. The PL (and $\mu$-PL) spectra shown in Figure $2 \mathrm{c}$ (and inset) demonstrate that up to 2.8-fold integrated intensity enhancement at RT is achieved in the QD-decorated GaN NW emission compared to that produced by bare GaN NWs (before QD drop-casting), whereas no peak shift is observed. It is worth noting the presence of a very weak yellow band indicates superior quality of the GaN NWs. To confirm this emission enhancement, we repeated RT $\mu$-PL measurements on QD-decorated NWs by recording the PL spectrum at different time intervals within the first three hours after the drop-casting process. The findings 
reported in Figure S2 in Supporting Information demonstrate that there is no significant change in the emission intensity of QD-decorated NWs over time, confirming the consistency of our results.

Figure 2d shows typical PL spectra produced by bare and QD-decorated NWs at low temperature $(5 \mathrm{~K})$ and at RT. At $5 \mathrm{~K}$, there are two dominant peaks; the most intense peak (Peak 1) is located at $\sim 3.47 \mathrm{eV}(357 \mathrm{~nm})$, which was attributed to the common GaN NBE, including free and bound exciton-related emissions. ${ }^{21,22}$ This peak is observed at both $5 \mathrm{~K}$ and RT (at $360 \mathrm{~nm}$ ). The second peak (Peak 2), located at $\sim 3.42 \mathrm{eV}(362 \mathrm{~nm})$ at $5 \mathrm{~K}$, is also a common GaN peak at low temperature and is dissociated at RT, likely due to excitons bound to stacking faults. ${ }^{21,22}$ As can be seen from Figure 2d, the PL emission of QD-decorated NWs is considerably enhanced at both RT and $5 \mathrm{~K}$, which is in line with the RT $\mu$-PL measurements.

The higher-resolution PL spectra provided in the inset of Figure $2 \mathrm{~d}$ confirm that the PL integrated intensities of Peak 1 and Peak 2 increase by $\sim 3.66$-fold and $\sim 3.9$-fold, respectively, after MnO QD drop-casting compared to bare GaN NWs. We attribute the greater enhancement at $5 \mathrm{~K}$ to the fact that the electron-hole recombination process is dominated by radiative recombination at low temperatures. These findings show that PL measurements indicate that, after QD functionalization, the NW emission increases by one order of magnitude compared to bare NWs, which is superior to the $<2$-fold enhancement reported for NWs surface-passivated by $\mathrm{KOH}$ solution. ${ }^{5}$ 

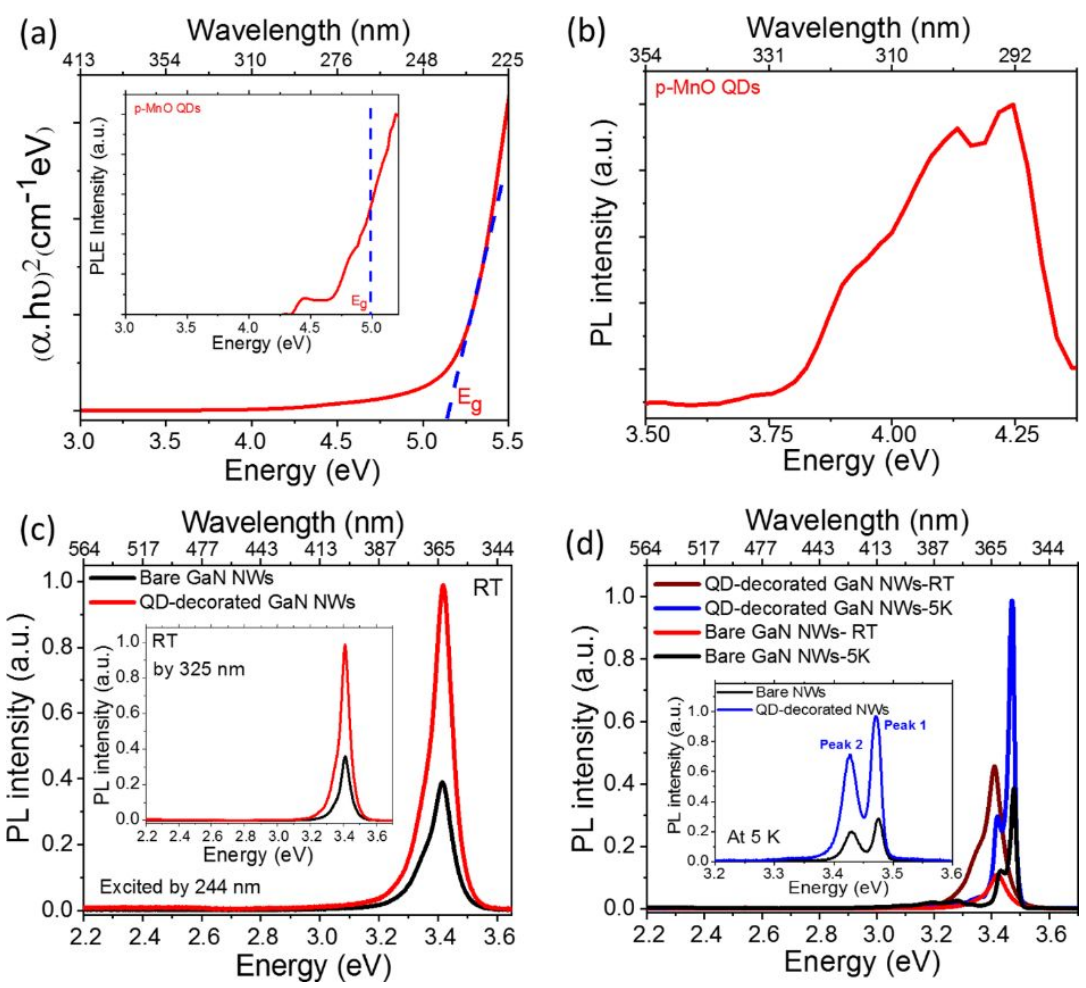

Figure 2. (a) Tauc absorption plot of MnO QDs in ethanol at RT. (The inset shows the corrected PLE spectra of MnO QDs in ethanol after subtracting the ethanol signal.) (b) The corrected PL spectrum of p-MnO QDs after subtracting the ethanol signal. (c) RT PL spectra of bare GaN NWs and QD-decorated NWs excited by $244 \mathrm{~nm}$. (The inset shows the RT $\mu$-PL spectra when excited by laser at $\lambda=325 \mathrm{~nm}$.) (d) Comparison of PL spectra at low temperature and RT for both bare GaN and QD-decorated GaN NWs (the inset shows higher-resolution PL spectra for both bare GaN and QD-decorated GaN NWs).

To rule out any laser or ethanol influence on the PL enhancement, we conducted detailed investigations of their effect on bare GaN NWs as a function of time. As shown in Figure S3a, the PL intensity of bare GaN (recorded at several time intervals) remained unchanged after prolonged laser exposure. Similarly, Figure S3b confirms that no significant change is observed in the GaN 
emission intensity after drop-casting ethanol only on bare NWs. Thus, these results confirm that QDs are solely responsible for enhancing the GaN emission.

The emission enhancement of QD-decorated NWs was optimized based on the QD density, as we observed that the GaN emission intensity decreased after several drop-casting cycles. $\mu$-PL measurements (shown in Figure 3a) reveal that the optimized density yields $\sim 2.8$-fold NBE intensity enhancement compared to that of bare NWs, which declines to $\sim 2$-fold after several cycles, and remains at this level thereafter. The FLAL synthesis method adopted in this work does not permit estimating the QD density after each cycle. However, the SEM image (Figure 3b) of NWs obtained after several drop-casting cycles reveals that when we performed more than three drop-casting cycles, we observed the formation of a densely packed QD-layer, and a few NWs were toppled over as can be seen in Figure $3 b$ due to drop-casting process. This could result in excessive scattering of emitted light, due to which only a small portion of emitted light can be collected. In addition, most likely, the QD clusters increase carrier scattering. Similar behavior has been observed in LEDs based on QDs; the emission intensity decreases as the QD density increases beyond certain threshold. For efficient transport, low-density QDs are preferable to welldistributed QDs, and can be obtained by both increasing the applied field, and by lowering the barrier between dots. ${ }^{20,23,24}$

To further elucidate the QD effect on GaN efficiency, we estimated the internal quantum efficiency (IQE) for both bare and QD-decorated NWs. In this case, the IQE represents the ratio of the integrated PL intensity of GaN NWs at $5 \mathrm{~K}$ to that at RT. We considered that maximum efficiency $(100 \%)$ is attained at $5 \mathrm{~K}$ by assuming that the non-radiative centers are frozen at low temperature (according to the Rashba's treatment). ${ }^{25-27}$ We estimated the IQE for NBE emission (Peak 1), as Peak 2 was absent from the PL spectra obtained at RT, as shown in Figure 2 d. The 
IQE value increases from $\sim 28 \%$ for bare GaN NWs to $\sim 46 \%$ when GaN NWs are hybridized with QDs, which confirms that radiative recombination contribution increases after QD functionalization. These results demonstrate that a considerable improvement in the $\mathrm{GaN}$ optical efficiency is achieved by functionalizing NWs with QDs.

To investigate the mechanisms underlying the observed emission enhancement, we performed PLE and ultrahigh-resolution EELS measurements on the QD-decorated GaN NW samples. Figure 4a shows the PLE spectra in close proximity to the NBE of GaN NWs (at $370 \mathrm{~nm}$ ) before and after QD drop-casting. It is evident that the GaN PLE spectrum is enhanced in the deep UV range (250-340 nm) after functionalizing the GaN NWs with QDs, while the GaN bandgap remains at $354 \mathrm{~nm}$. The greatest PLE signal enhancement ( 2-fold) occurs at $>250 \mathrm{~nm}(4.95 \mathrm{eV}$, which is close to the QD bandgap). This value is similar to the enhancement ratio of the PL integrated peak intensity after functionalizing NWs with QDs.
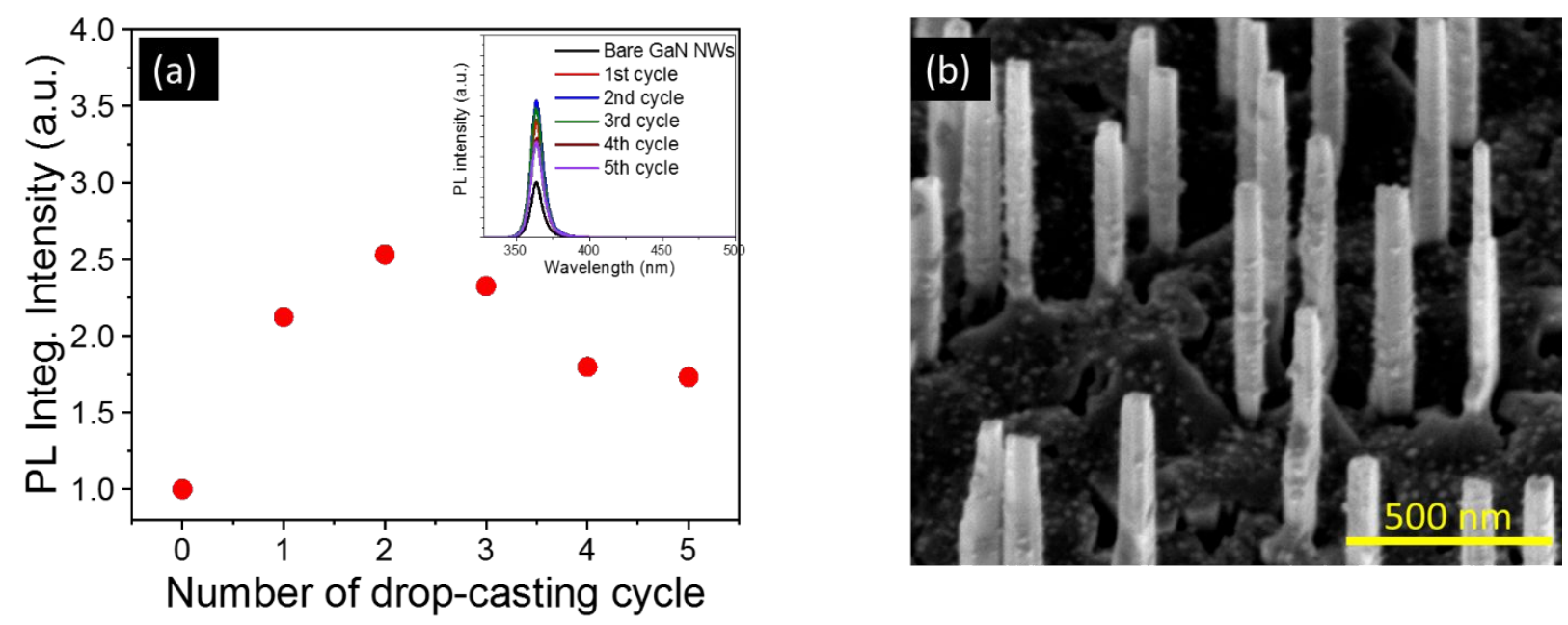

Figure 3. (a) PL intensity of GaN NWs as function of QD density (drop-casting cycles) and the inset shows the corresponding PL spectra and SEM image of GaN NWs after three drop-casting cycles of $\mathrm{MnO}$ in ethanol. 

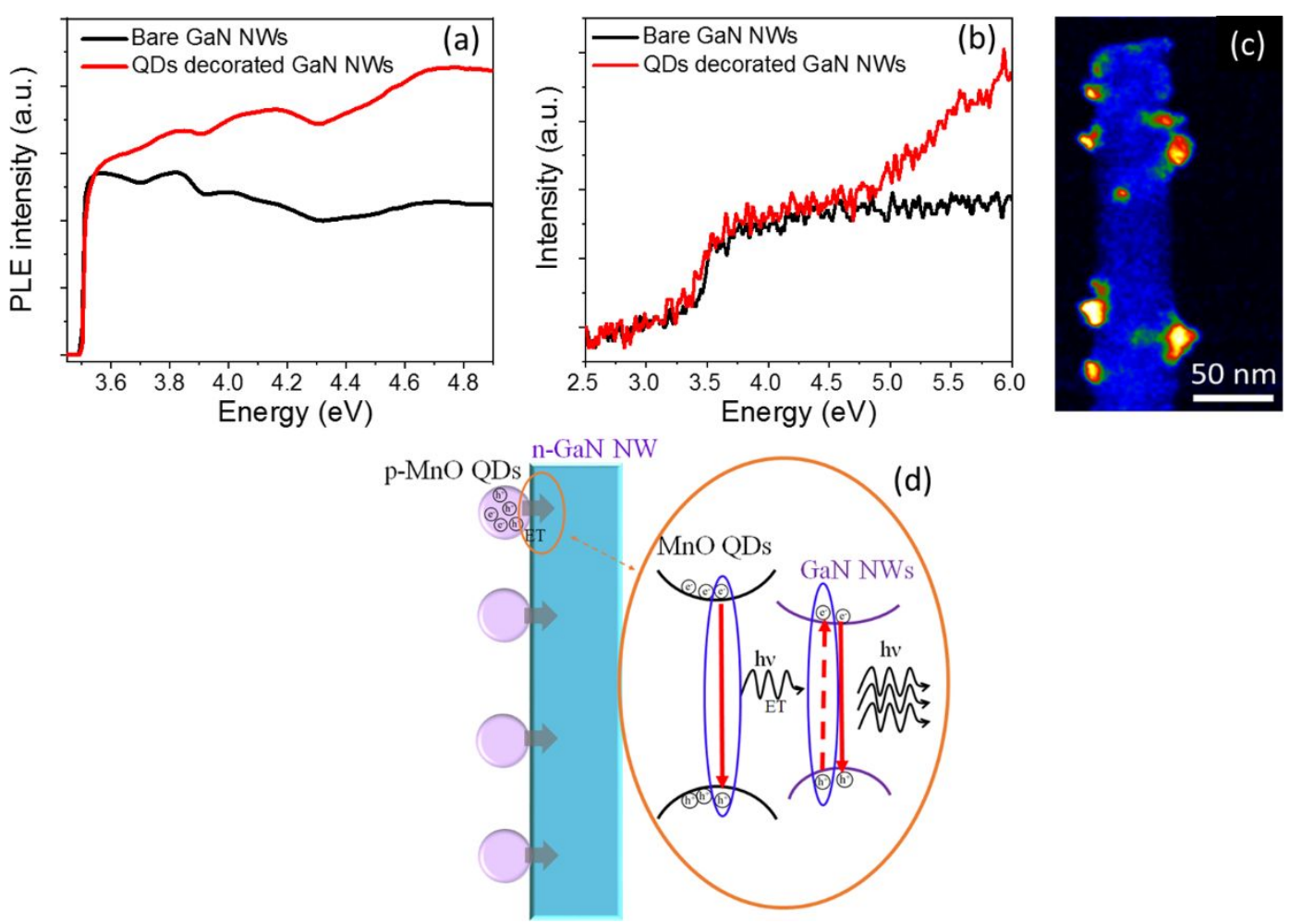

Figure 4. (a) PLE spectra of bare GaN NWs and those decorated with QDs. (b) Comparison of ultrahigh-resolution low-loss EELS signal (after background subtraction) obtained from bare GaN NWs with that produced by GaN NWs decorated with QDs. (c) Fitting map of the low-loss EELS signal obtained from GaN NWs vs. signal produced by QDs. (d) Schematic diagram of ET from MnO QDs to GaN NWs.

Ultrahigh-resolution EELS measurements at a very low loss energy $(200 \mathrm{meV})$ were also conducted and the results are shown in Figure 4b. For this purpose, the spatial variation of the bandgap was mapped with the energy resolution of about $45-50 \mathrm{meV}$, defined as the full width at half maximum (FWHM) of the zero energy loss peak (ZLP). ${ }^{21,}{ }^{22}$ Moreover, the background subtraction was performed by fitting the ZLP measured in a vacuum. The ultrahigh-resolution EELS analysis demonstrates that QDs absorb some energy, as indicated by "glowing" QDs in the 
EELS map in Figure 4c. The EELS signal in the energy range above the bandgap produced by bare GaN differs from that obtained for QD-decorated GaN NWs. Specifically, the signals shown in Figure $4 \mathrm{~b}$ indicate that, for both bare NWs and QD-decorated NWs, the onset of the conduction band transition (bandgap width) starts at about the same energy level $(\sim 3.4 \mathrm{eV})$, corresponding to the NW bandgap, ${ }^{18}$ which is in line with the PLE results. However, at $4.95 \mathrm{eV}$ (corresponding to $\lambda=250 \mathrm{~nm}$, which is close to the QD bandgap) the signal grows faster for QD-decorated NWs compared to the bare ones. The EELS findings indicate a significant increase in the density of states of the QD-decorated GaN NWs due to QD functionalization, compared to bare GaN NWs, ${ }^{21}$, 22 thus confirming the PL and PLE results. It is worth noting that, as expected, no plasmonic effect has been observed by EELS, as the QDs are wide-bandgap semiconductor material.

The energy band diagram of the QD-decorated GaN sample is shown in Figure S4 in Supporting Information, confirming a good bandgap overlap between GaN and p-MnO QDs. Moreover, based on the PLE and EELS findings, the PL enhancement of QD-decorated NWs can be attributed to the energy transfer from MnO QDs to GaN due to the bandgap difference. In this case, for QD-decorated NWs, after exciting QDs and generating electron-hole $(\mathrm{e}-\mathrm{h})$ pairs, the energy resulting from $\mathrm{e}-\mathrm{h}$ recombination process is transferred to the $\mathrm{GaN} \mathrm{NW}$ band to excite more electrons from the valence band to the conduction band, thus increasing the $\mathrm{e}^{-} \mathrm{h}$ pair density (i.e., the ET process occurred from a higher to a lower bandgap energy, as shown in Figure 4d). In this case, the GaN carriers are excited directly by laser as well as via ET process, resulting in enhancement of the radiative recombination processes ${ }^{18}$ compared to bare GaN NWs, as the band alignment (shown in Figure S4) facilitates such ET mechanism. Specially, there is energy resonance between the bandedge tail (shown below the $\mathrm{MnO}$ bandedge) and the GaN bandedge, as indicated by the PLE and absorption measurements of MnO QDs. The non-radiative ET 
phenomenon has been observed, but such ET direction was reverse (i.e., from III-nitride material to smaller-bandgap QDs), ${ }^{28-30}$ facilitating development of visible LED. However, ET from widerbandgap QDs to GaN has never been reported.

To elucidate our ET transfer hypothesis, we carried out time-resolved PL (TRPL) measurements before and after functionalizing NWs by QDs. We analyzed the carrier lifetime $\left(\tau_{\mathrm{PL}}\right)$ for Peak 2 of GaN NWs at $5 \mathrm{~K}$, as this peak emerges at low temperature, suggesting that the radiative-recombination process is dominant in this spectral range. In addition, as noted earlier, it was assumed that most non-radiative recombination centers are frozen at low temperature. ${ }^{25,26,28,}$ ${ }^{29,31}$ As shown in Figure S5 in Supporting Information, $\tau_{\mathrm{PL}}$ of Peak 2 decreases from 2.03 ns (for bare GaN NWs) to $1.39 \mathrm{~ns}$ (for QD-decorated $\mathrm{GaN}$ ), resulting in a $\sim 0.68 \tau_{\mathrm{PL}}$ ratio, from which it can be inferred that the radiative recombination rate increases after QDs functionalization..$^{29,32}$

To further confirm our ET hypothesis, we functionalized $\mathrm{ZnO}$ nanotubes (NTs) grown on $\mathrm{Si}(\sim 3.7 \mathrm{eV}$ bandgap) by MnO QDs using the same drop-casting process (the growth process and the properties of these NTs are reported elsewhere ${ }^{18}$ using pulsed laser deposition (PLD)). ${ }^{33,34}$ As shown in Figure S6, the emission intensity increases by $>$ 4-fold, providing evidence in support of our assertion that depositing wider-bandgap QDs on the surface of a smaller-bandgap semiconductor nanostructures can lead to semiconductor enhancement. As this is a novel study, additional investigations may be needed to elucidate the underlying mechanisms. Nonetheless, this novel finding has important implications for future efforts aimed at utilizing wide-bandgap QD materials to obtain devices operating in the deep UV range. We expect that, in QD-decorated NWbased LED structure, the turn-on voltage is reduced due to greater carrier density resulting in higher EL intensity compared to that from bare NWs. In addition, anti-reflective coating may be 
needed to further improve further the light extracting efficiency, leading to high efficiency UV light emitting devices. ${ }^{15,35}$

\section{Conclusions}

In this work, we reported on the first study as a part of which the optical UV efficiency of NWs was enhanced significantly by functionalizing them by p-MnO QDs characterized by a wider bandgap to transfer the energy from the QDs to GaN. Our findings show that, when highly crystalline p-MnO QDs are drop-casted on GaN NWs, the NBE emission of GaN is significantly ( $\sim 290 \%$ ) enhanced, i.e., it is $\sim 3.9$-fold greater than that produced by bare GaN NWs. A considerable IQE increase obtained after decorating GaN with QDs further demonstrates that the radiative recombination rate increases after decorating NWs with QDs. As this process has never been used or studied for enhancing III-nitride emission, we have succeeded in demonstrating that functionalizing QDs (with $5 \mathrm{eV}$ bandgap) with III-nitrides (including GaN, InGaN or AlGaN NWs) has the potential to enhance the LED efficiency. EELS and PLE measurements showed enhancement in the deep UV range after functionalizing the GaN NWs, while also revealing that, above $4.9 \mathrm{eV}$ (equivalent to the QD bandgap), the signal grows faster for QD-decorated NWs above, indicating an increase in the density of states after functionalizing GaN with QDs. These analyses show that the energy transfer occurs from $\mathrm{MnO}$ to GaN NWs, which is the most likely reason behind the GaN emission enhancement. Thus, this novel environmentally-friendly (with zero waste) strategy would be highly beneficial for state-of-the-art technology development aimed at enhancing the performance of light emitting devices, as well as in wide-bandgap semiconductor applications, such as transistors, photovoltaic cells, and photodetectors. 


\section{ASSOCIATED CONTENT \\ Supporting Information}

The PL and PLE spectra of ethanol; The PL emissions of QDs-decorated NWs and bare NWs as a function of time to investigate the laser effect and ethanol effect; TRPL measurements; The enhancement of PL emission from MnO QD-decorated $\mathrm{ZnO}$ nanotubes.

ACS Publications website at DOI:

AUTHOR INFORMATION

Corresponding Authors

*E-mail: iman.roqan@kaust.edu.sa.

\section{Author Contributions}

Dhaifallah Almalawi and Iman S Roqan have equal first author contribution. All authors have given approval to the final version of the manuscript.

\section{Notes}

The authors declare no competing financial interest.

\section{ACKNOWLEDGMENTS}

Authors thanks KAUST for the finance support using the base fund number BAS/1/1319-01-01.

\section{References}

1. Heilmann, M.; Munshi, A. M.; Sarau, G.; Göbelt, M.; Tessarek, C.; Fauske, V. T.; van Helvoort, A. T.; Yang, J.; Latzel, M.; Hoffmann, B. r., Vertically oriented growth of GaN nanorods on Si using graphene as an atomically thin buffer layer. Nano letters 2016, 16 (6), 35243532. 
2. Jones, E. A.; Wang, F. F.; Costinett, D., Review of commercial GaN power devices and GaN-based converter design challenges. IEEE Journal of Emerging and Selected Topics in Power Electronics 2016, 4 (3), 707-719.

3. Lin, T.-N.; Santiago, S. R. M.; Yuan, C.-T.; Chiu, K.-P.; Shen, J.-L.; Wang, T.-C.; Kuo, H.-C.; Chiu, C.-H.; Yao, Y.-C.; Lee, Y.-J., Enhanced performance of GaN-based ultraviolet light emitting diodes by photon recycling using graphene quantum dots. Scientific reports $\mathbf{2 0 1 7 ,} 7$ (1), 7108.

4. Kim, B.-J.; Yang, G.; Kim, H.-Y.; Baik, K. H.; Mastro, M. A.; Hite, J. K.; Eddy, C. R.; Ren, F.; Pearton, S. J.; Kim, J., GaN-based ultraviolet light-emitting diodes with AuCl 3-doped graphene electrodes. Optics express 2013, 21 (23), 29025-29030.

5. Sun, H.; Shakfa, M. K.; Muhammed, M. M.; Janjua, B.; Li, K.-H.; Lin, R.; Ng, T. K.; Roqan, I. S.; Ooi, B. S.; Li, X., Surface-passivated AlGaN nanowires for enhanced luminescence of ultraviolet light emitting diodes. Acs Photonics 2017, 5 (3), 964-970.

6. Liu, C.; Ooi, Y. K.; Islam, S.; Verma, J.; Xing, H.; Jena, D.; Zhang, J., Physics and polarization characteristics of $298 \mathrm{~nm}$ AlN-delta-GaN quantum well ultraviolet light-emitting diodes. Applied Physics Letters 2017, 110 (7), 071103.

7. Khan, A.; Balakrishnan, K.; Katona, T., Ultraviolet light-emitting diodes based on group three nitrides. Nature photonics 2008, 2 (2), 77.

8. Zhao, S.; Connie, A.; Dastjerdi, M.; Kong, X.; Wang, Q.; Djavid, M.; Sadaf, S.; Liu, X.; Shih, I.; Guo, H., Aluminum nitride nanowire light emitting diodes: Breaking the fundamental bottleneck of deep ultraviolet light sources. Scientific reports 2015, 5, 8332.

9. Djavid, M.; Mi, Z., Enhancing the light extraction efficiency of AlGaN deep ultraviolet light emitting diodes by using nanowire structures. Applied Physics Letters 2016, 108 (5), 051102. 
10. Alfaraj, N.; Mitra, S.; Wu, F.; Ajia, I. A.; Janjua, B.; Prabaswara, A.; Aljefri, R. A.; Sun, H.; Khee Ng, T.; Ooi, B. S., Photoinduced entropy of InGaN/GaN pin double-heterostructure nanowires. Applied Physics Letters 2017, 110 (16), 161110.

11. AlOtaibi, B.; Harati, M.; Fan, S.; Zhao, S.; Nguyen, H.; Kibria, M.; Mi, Z., High efficiency photoelectrochemical water splitting and hydrogen generation using GaN nanowire photoelectrode. Nanotechnology 2013, 24 (17), 175401.

12. Hui, T. S.; Zaini, M. A. A., Potassium hydroxide activation of activated carbon: a commentary. Carbon Letters (Carbon Lett.) 2015, 16 (4), 275-280.

13. Wang, S.; Dai, J.; Hu, J.; Zhang, S.; Xu, L.; Long, H.; Chen, J.; Wan, Q.; Kuo, H.-c.; Chen, C., Ultrahigh degree of optical polarization above $80 \%$ in AlGaN-based deep-ultraviolet LED with moth-eye microstructure. ACS Photonics 2018, 5 (9), 3534-3540.

14. Kapoor, A.; Finot, S.; Grenier, V.; Robin, E.; Bougerol, C.; Bleuse, J.; Jacopin, G.; Eymery, J.; Durand, C., Role of underlayer for efficient core-shell InGaN QWs grown on m-plane GaN wire sidewalls. ACS Applied Materials \& Interfaces 2020.

15. Jung, J.-Y.; Zhou, K.; Bang, J. H.; Lee, J.-H., Improved photovoltaic performance of Si nanowire solar cells integrated with ZnSe quantum dots. The Journal of Physical Chemistry C 2012, $116(23), 12409-12414$.

16. Mitra, S.; Aravindh, A.; Das, G.; Pak, Y.; Ajia, I.; Loganathan, K.; Di Fabrizio, E.; Roqan, I. S., High-performance solar-blind flexible deep-UV photodetectors based on quantum dots synthesized by femtosecond-laser ablation. Nano Energy 2018, 48, 551-559.

17. Mitra, S.; Pak, Y.; Alaal, N.; Hedhili, M. N.; Almalawi, D. R.; Alwadai, N.; Loganathan, K.; Kumarasan, Y.; Lim, N.; Jung, G. Y., Novel P-Type Wide Bandgap Manganese Oxide 
Quantum Dots Operating at Deep UV Range for Optoelectronic Devices. Advanced Optical Materials 2019.

18. Govyadinov, A. A.; Konečná, A.; Chuvilin, A.; Vélez, S.; Dolado, I.; Nikitin, A. Y.; Lopatin, S.; Casanova, F.; Hueso, L. E.; Aizpurua, J., Probing low-energy hyperbolic polaritons in van der Waals crystals with an electron microscope. Nature communications 2017, 8 (1), 95.

19. Lopatin, S.; Cheng, B.; Liu, W.-T.; Tsai, M.-L.; He, J.-H.; Chuvilin, A., Optimization of monochromated TEM for ultimate resolution imaging and ultrahigh resolution electron energy loss spectroscopy. Ultramicroscopy 2018, 184, 109-115.

20. Pak, Y.; Mitra, S.; Alaal, N.; Xin, B.; Lopatin, S.; Almalawi, D.; Min, J.-W.; Kim, H.; Kim, W.; Jung, G.-Y., Dark-current reduction accompanied photocurrent enhancement in p-type MnO quantum-dot decorated n-type 2D-MoS2-based photodetector. Applied Physics Letters 2020, $116(11), 112102$.

21. Paskov, P.; Schifano, R.; Monemar, B.; Paskova, T.; Figge, S.; Hommel, D., Emission properties of a-plane GaN grown by metal-organic chemical-vapor deposition. Journal of Applied Physics 2005, 98 (9), 093519.

22. Liu, R.; Bell, A.; Ponce, F.; Chen, C.; Yang, J.; Khan, M. A., Luminescence from stacking faults in gallium nitride. Applied Physics Letters 2005, 86 (2), 021908.

23. Fauchet, P. M., Light emission from Si quantum dots. Materials Today 2005, 8 (1), 26-33. 24. Liu, S.-M.; Guo, H.-Q.; Zhang, Z.-H.; Li, R.; Chen, W.; Wang, Z.-G., Characterization of $\mathrm{CdSe}$ and $\mathrm{CdSe} / \mathrm{CdS}$ core/shell nanoclusters synthesized in aqueous solution. Physica E: Lowdimensional Systems and Nanostructures 2000, 8 (2), 174-178.

25. Brandt, O.; Ringling, J.; Ploog, K. H.; Wünsche, H.-J.; Henneberger, F., Temperature dependence of the radiative lifetime in GaN. Physical Review B 1998, 58 (24), R15977. 
26. Rashba, E., Giant oscillator-strengths associated with exciton complexes. SOVIET PHYSICS SEMICONDUCTORS-USSR 1975, 8 (7), 807-816.

27. Flemban, T. H.; Sequeira, M.; Zhang, Z.; Venkatesh, S.; Alves, E.; Lorenz, K.; Roqan, I. S., Identifying the influence of the intrinsic defects in Gd-doped $\mathrm{ZnO}$ thin-films. Journal of Applied Physics 2016, 119 (6), 065301.

28. Smith, R.; Liu, B.; Bai, J.; Wang, T., Hybrid III-nitride/organic semiconductor nanostructure with high efficiency nonradiative energy transfer for white light emitters. Nano letters 2013, 13 (7), 3042-3047.

29. Nizamoglu, S.; Sari, E.; Baek, J.-H.; Lee, I.-H.; Demir, H. V., White light generation by resonant nonradiative energy transfer from epitaxial $\mathrm{InGaN} / \mathrm{GaN}$ quantum wells to colloidal CdSe/ZnS core/shell quantum dots. New Journal of Physics 2008, 10 (12), 123001.

30. Zhang, F.; Liu, J.; You, G.; Zhang, C.; Mohney, S. E.; Park, M. J.; Kwak, J. S.; Wang, Y.; Koleske, D. D.; Xu, J., Nonradiative energy transfer between colloidal quantum dot-phosphors and nanopillar nitride LEDs. Optics express 2012, 20 (102), A333-A339.

31. Muhammed, M. M.; Alwadai, N.; Lopatin, S.; Kuramata, A.; Roqan, I. S., HighEfficiency InGaN/GaN Quantum Well-Based Vertical Light-Emitting Diodes Fabricated on $\beta$ Ga2O3 Substrate. ACS applied materials \& interfaces 2017, 9 (39), 34057-34063.

32. Ajia, I. A.; Yamashita, Y.; Lorenz, K.; Muhammed, M.; Spasevski, L.; Almalawi, D.; Xu, J.; Iizuka, K.; Morishima, Y.; Anjum, D. H., GaN/AlGaN multiple quantum wells grown on transparent and conductive (-201)-oriented $\beta$-Ga2O3 substrate for UV vertical light emitting devices. Applied Physics Letters 2018, 113 (8), 082102. 
33. Venkatesh, S.; Franklin, J.; Ryan, M.; Lee, J.-S.; Ohldag, H.; McLachlan, M.; Alford, N.; Roqan, I. S., Defect-band mediated ferromagnetism in Gd-doped $\mathrm{ZnO}$ thin films. Journal of Applied Physics 2015, 117 (1), 013913.

34. Roqan, I. S.; Venkatesh, S.; Zhang, Z.; Hussain, S.; Bantounas, I.; Franklin, J.; Flemban, T. H.; Zou, B.; Lee, J.-S.; Schwingenschlogl, U., Obtaining strong ferromagnetism in diluted Gddoped $\mathrm{ZnO}$ thin films through controlled Gd-defect complexes. Journal of Applied Physics 2015, $117(7), 073904$.

35. Cho, J.-Y.; Byeon, K.-J.; Lee, H., Forming the graded-refractive-index antireflection layers on light-emitting diodes to enhance the light extraction. Optics letters 2011, 36 (16), 32033205. 
Table of contents:

Enhanced UV Emission of GaN Nanowires Functionalized by Wider Bandgap SolutionProcessed p-MnO Quantum Dots

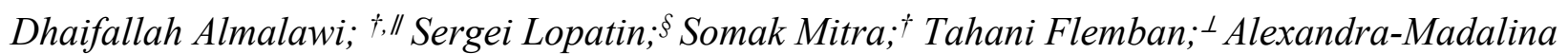
Siladie, $;$ Bruno Gayral; $;$ Bruno Daudin; $;$ and Iman S. Roqan ${ }^{\dagger * *}$

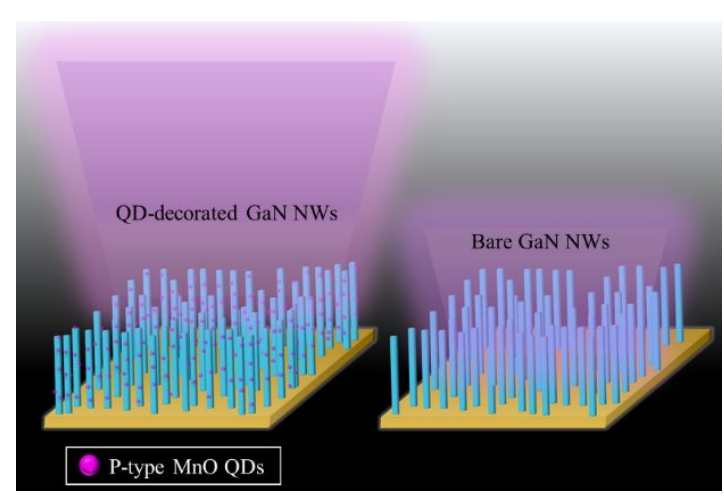




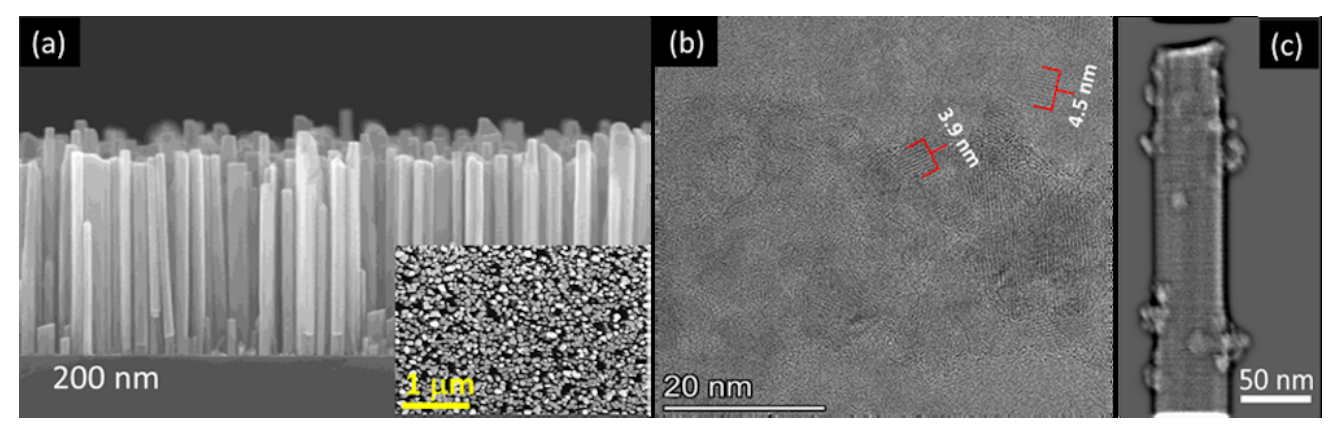

Figure 1

$85 \times 26 \mathrm{~mm}(300 \times 300 \mathrm{DPI})$ 
(a) Wavelength ( $\mathrm{nm}$ )

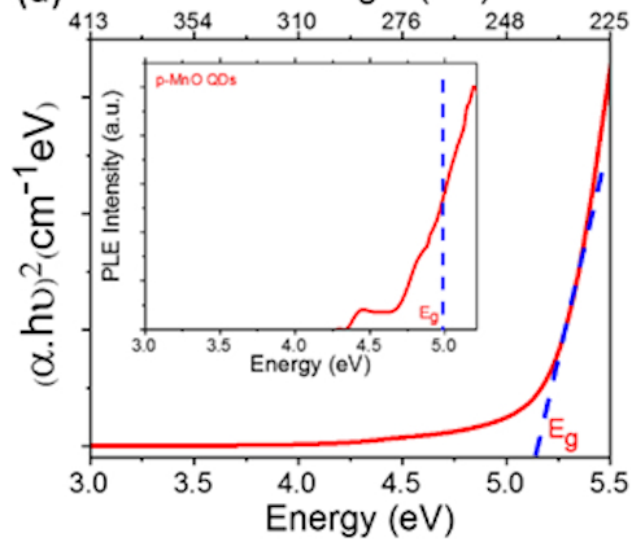

(c) Wavelength $(\mathrm{nm})$

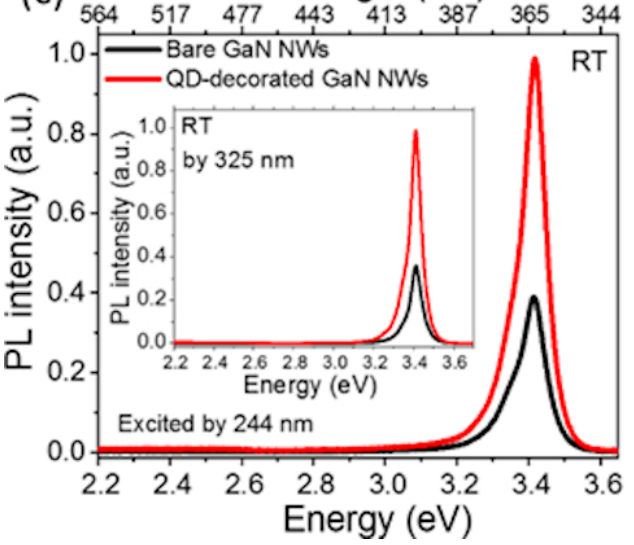

(b)

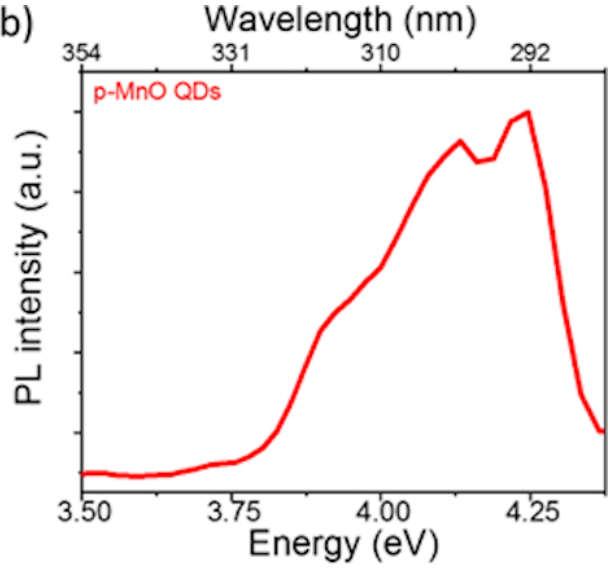

Wavelength $(\mathrm{nm})$

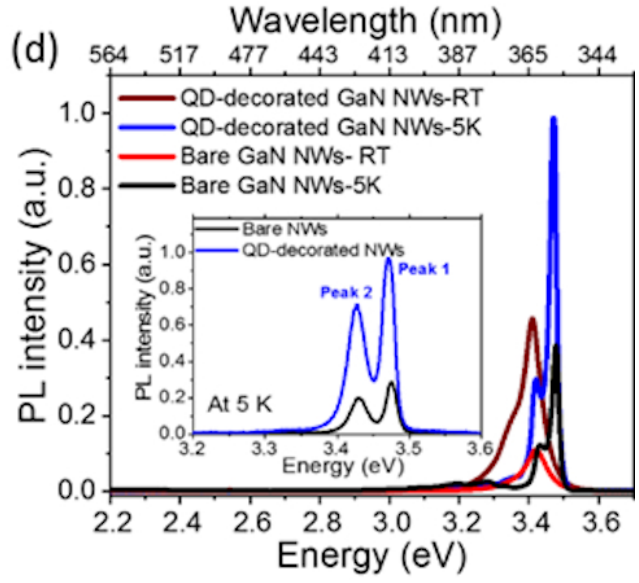

Figure 2

$85 \times 76 \mathrm{~mm}(300 \times 300 \mathrm{DPI})$ 

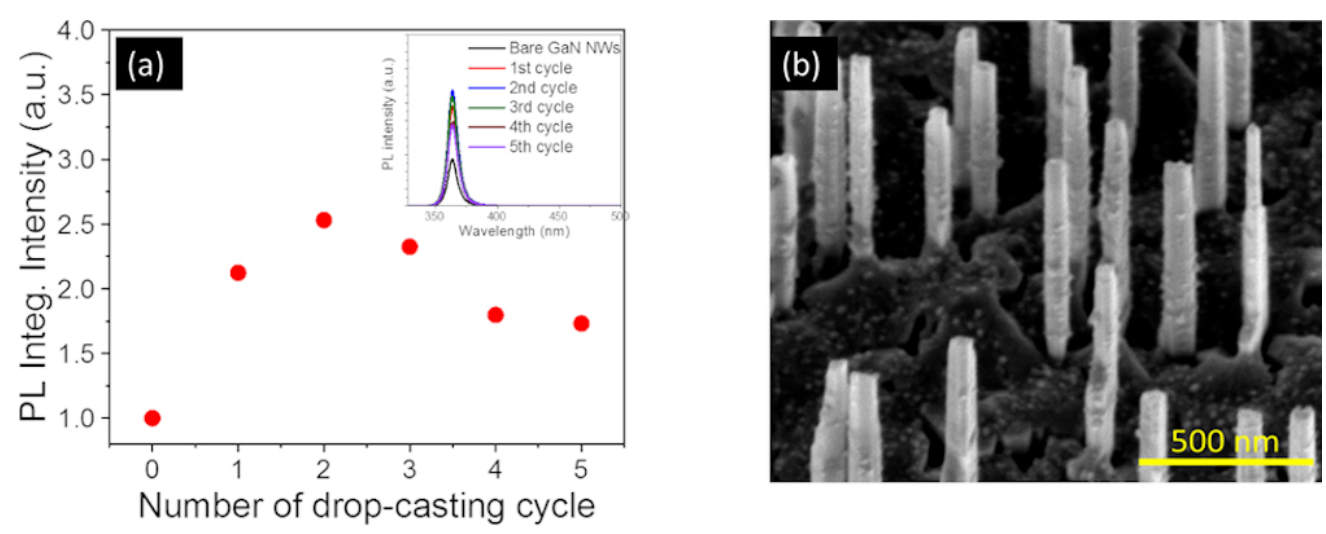

Figure 3

$85 \times 33 \mathrm{~mm}(300 \times 300 \mathrm{DPI})$ 

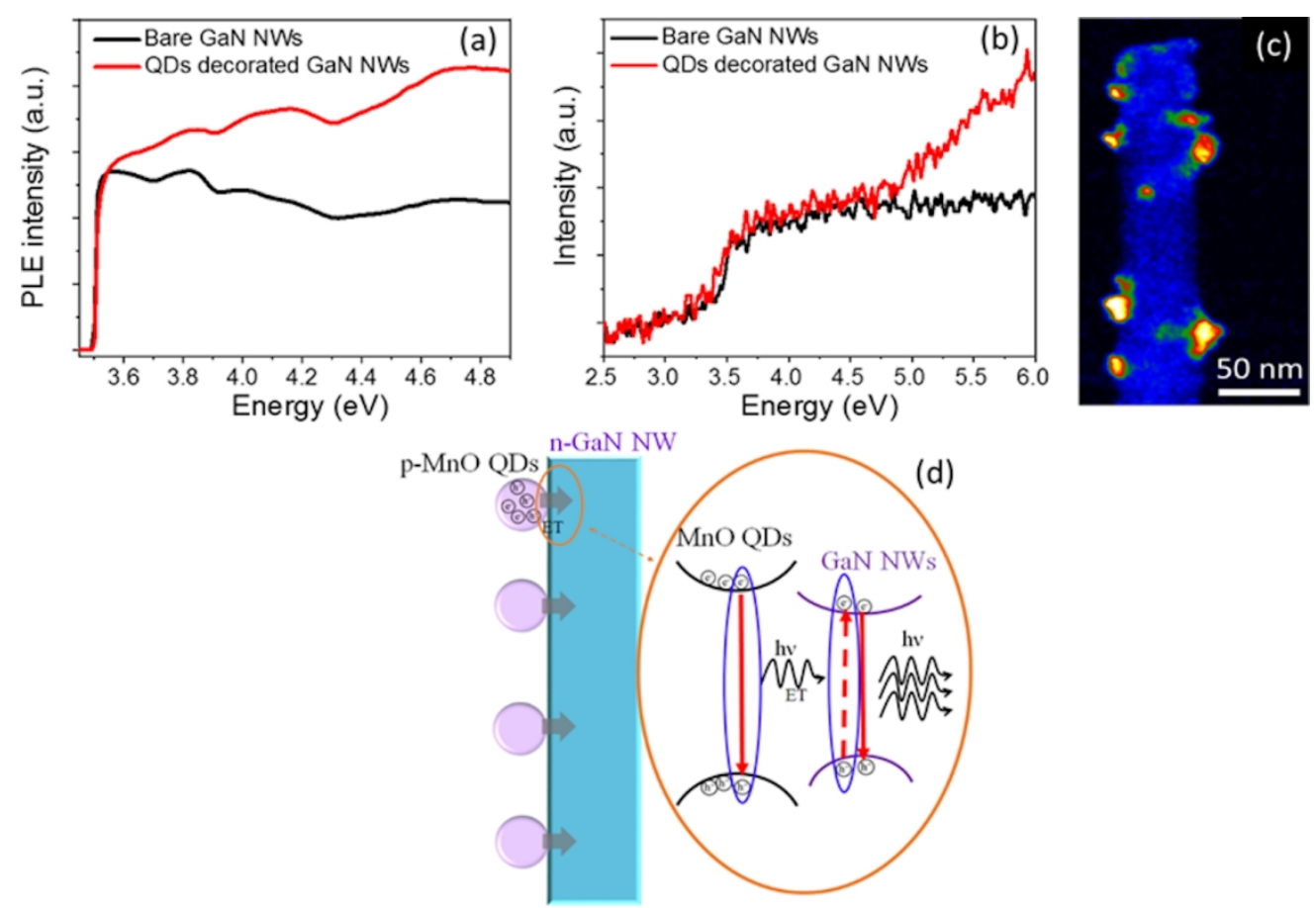

Figure 4

$129 \times 89 \mathrm{~mm}(300 \times 300$ DPI $)$ 


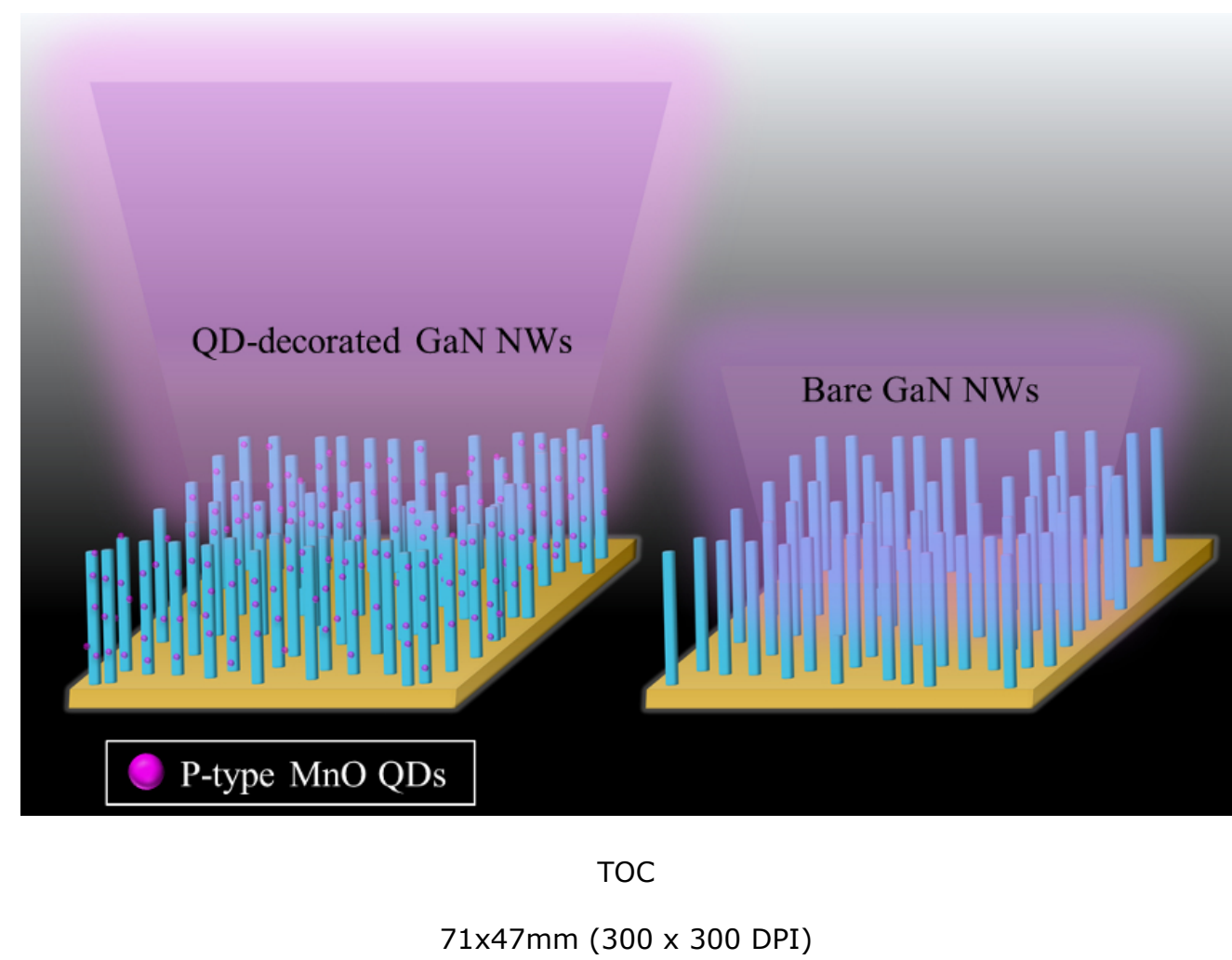

